hep-th/0307179

DAMTP-2003-68

\title{
Inflationary Cosmologies from Compactification?
}

\author{
Mattias N.R. Wohlfarth* \\ Department of Applied Mathematics and Theoretical Physics, \\ Centre for Mathematical Sciences, University of Cambridge, \\ Wilberforce Road, Cambridge CB3 0WA, U.K.
}

\begin{abstract}
We consider the compactification of $(d+n)$-dimensional pure gravity and of superstring/M-theory on an $n$-dimensional internal space to a $d$-dimensional FLRW cosmology, with spatial curvature $k=0, \pm 1$, in Einstein conformal frame. The internal space is taken to be a product of Einstein spaces, each of which is allowed to have arbitrary curvature and a time-dependent volume. By investigating the effective $d$-dimensional scalar potential, which is a sum of exponentials, it is shown that such compactifications, in the $k=0,+1$ cases, do not lead to large amounts of accelerating expansion of the scale factor of the resulting FLRW universe, and, in particular, not to inflation. The case $k=-1$ admits solutions with eternal accelerating expansion for which the acceleration, however, tends to zero at late times.
\end{abstract}

PACS numbers: $11.25 . \mathrm{Mj}, 98.80 . \mathrm{Cq}, 04.50 .+\mathrm{h}$

*Electronic address: M.N.R.Wohlfarth@damtp.cam.ac.uk 


\section{INTRODUCTION}

The relationship between cosmology and superstring or M-theory is, at least, twofold. On the one hand, cosmology greatly benefits from the existence of an underlying, and consistent, unified theory of fundamental particle interactions and of gravity. The hope may be expressed that the cosmology of our universe will one day become derivable from higher dimensions. On the other hand, it is well known that high energy phenomena near the Planck scale, which are described by string/M-theory, are almost inaccessible to local observations. Therefore, cosmological observations, influenced by physics on an enormous range of scales, become probably the most important testing ground for these theories.

One particular aspect of cosmology, based on recent observations of supernovae and the microwave background, is the emerging evidence for an accelerating expansion of the universe. Not only a present epoch of accelerating expansion is indicated, but also an inflationary epoch in the distant past. Much attention has been paid recently to the construction of cosmological solutions, from compactifications of string/M-theory, which exhibit such behaviour. The possibility of having, at least one period of, accelerating expansion of the FLRW scale factor has first been demonstrated in [1], thereby contradicting the general belief that this was impossible, which was motivated by the difficulties to find maximally symmetric de Sitter solutions from compactifications of supergravity theories and by a no-go theorem for such compactifications [2].

The work of [1] was done in the context of pure gravity. Subsequently, it was generalised to include fluxes, as are necessary to consider in string/M-theory compactifications, and, furthermore, a connection to S-branes was established [3] (see references to the S-brane literature therein). The original solution (in eleven dimensions), in fact, turns out to be the zero flux limit of an electrically charged SM2-brane solution. An investigation into the properties of the phase of accelerating expansion in these solutions, performed in [4], revealed the fact that this phase is very short in the sense that it only allows a fixed amount of expansion of the universe, independent of the parameters. The expansion factor turned out to be about two, i.e., of an order of magnitude $\mathcal{O}(1)$, and a similar analysis suggests

that this is not improved in other examples [5]. So the known solutions do not apply to inflationary scenarios. They might, however, according to [6], turn out to be useful for describing present day acceleration. 
An intuitive explanation for the short accelerating phase and the resulting smallness of the expansion factor was given in [7] from the four-dimensional point of view (we consider $d+n$ dimensions here). The compactification produces scalar fields as moduli of the metric of the internal space. The effective lower-, $d$-dimensional theory, written down for the case of a single scalar $\phi$, is of the generic form

$$
S=\int \sqrt{-g}\left[\frac{1}{2} R-(\nabla \phi)^{2}-2 V(\phi)\right] .
$$

Considering FLRW solutions for the metric, i.e.,

$$
d s^{2}=-d t^{2}+a(t)^{2} d \Sigma_{k}^{2}
$$

where $k=0, \pm 1$ denotes the curvature of the $(d-1)$-dimensional spatial sections, it now follows from the equations of motion of the above action that the scale factor can exhibit accelerating expansion only if the potential of the scalar field is larger than its kinetic energy and satisfies,

$$
V>\frac{d-2}{2} \dot{\phi}^{2}
$$

In the cosmological compactifications discussed above, the scalar field starts out at infinite value but with high kinetic energy. It runs into a positive and steep exponential potential, turns around and falls back. At the turning point, the solutions are dominated by potential energy and, consequently, the scale factor accelerates. But this phase cannot last long because of the steepness of the exponential potential.

The acceleration condition (31) is easily extended to the case of several scalar fields by summing their kinetic energies on the right hand side. It presents a very simple criterion that has to be satisfied in order to find solutions with accelerating expansion. Note that a necessary requirement for this purpose is to have positive potentials.

An almost too easy way to satisfy the acceleration condition, which we do not further discuss here, is opened up by so called phantom scalar fields, see e.g. [8], motivated perhaps from the starred string theories that are obtained from the conventional ones by timelike T-duality [9]. These phantom fields have a different sign in their kinetic energy term, and, replacing $\dot{\phi}^{2} \mapsto-\dot{\phi}^{2}$, one finds that the acceleration condition is always satisfied when the potential is positive and possibly also when it is negative.

Another way of extending the known results on accelerating cosmologies has been followed in [10,11], where the authors find new solutions by considering compactifications on product 
spaces, in which each factor is provided with its own volume modulus. They do not find improved properties of the accelerating phase, but they make an important observation. So far the discussion has assumed $k=0$. If negative curvature is introduced on the spatial sections of the cosmology, a solution with critical scale factor, i.e., with $a \sim t$, can be found and a perturbation around this solution may lead to eternal acceleration.

The plan of this paper is to embed all the results and observations on accelerating cosmologies obtained from compactifications of string/M-theory into a single coherent point of view. To achieve such an understanding, we consider the effective lower-dimensional theories and investigate the generic properties of the resulting potential for the scalar fields. We do not have to solve equations of motion, and do not present any new solutions, but our discussion will show clearly which types of solutions may exist. Highlighting examples are taken from previous papers. A question of particular interest will be whether there are solutions with inflationary behaviour.

Section II considers attractor solutions for the scale factor of an FLRW cosmology in the system of gravity coupled to a single scalar field moving in an exponential potential. These are related to the characteristic exponent of the potential. We argue that the case of many scalar fields can effectively be reduced to the single scalar case and calculate the characteristic exponents for more complicated potentials. The basic dimensional reduction of $(d+n)$-dimensional gravity is performed in section III. The potential for the scalar fields is determined, and its consequences for possible cosmologies are discussed. Section IV extends this discussion to string/M-theory compactifications by including relevant fluxes and the dilaton field. Section $\mathrm{V}$ presents the mechanism of consistent truncation which reduces the number of scalar fields and might possibly improve on our results. We conclude with a discussion in section VI.

\section{ATTRACTOR SOLUTIONS}

Before compactifying $(d+n)$-dimensional pure gravity or string/M-theory on product spaces down to $d$ dimensions, as we will do in sections III and IV let us first discuss a few features of the arising lower-dimensional theory. For the scalar fields we will find potentials that are sums of exponential terms. It will, however, turn out that, in order to understand the case of many scalar fields, it is sufficient to understand the case of a single scalar field. So 


\begin{tabular}{c||c|c|c|c}
$k \cdot \cdot \alpha^{2}$ & $0<\alpha^{2}<\alpha_{c}^{2}$ & $\alpha^{2}=\alpha_{c}^{2}$ & $\alpha_{c}^{2}<\alpha^{2}<\alpha_{h}^{2}$ & $\alpha_{h}^{2} \leq \alpha^{2}$ \\
\hline \hline-1 & $t^{\left(\alpha_{c} / \alpha\right)^{2}}$ & $t$ & $t$ & $t$ \\
\hline 0 & $t^{\left(\alpha_{c} / \alpha\right)^{2}}$ & $t$ & $t^{\left(\alpha_{c} / \alpha\right)^{2}}$ & $t^{\left(\alpha_{c} / \alpha_{h}\right)^{2}}$ \\
\hline+1 & $t^{\left(\alpha_{c} / \alpha\right)^{2}}$ & $*$ & $*$ & $*$
\end{tabular}

Table I: The scale factor for attractor solutions in exponential scalar potentials for $k=0, \pm 1$ and various ranges of $\alpha^{2}$. The $k=+1$ cases marked with an asterisk undergo recollapse at late times and do not have attractor solutions. Before that, they may, however, behave very much like the $k=0$ solutions.

let us for now restrict our attention to the action (11) and consider an exponential potential

$$
V(\phi)=\Lambda e^{-2 \alpha \phi}
$$

When the potential is positive with $\Lambda>0$, the system of gravity coupled to such a scalar field exhibits cosmological late-time attractor solutions, see e.g. [12]. For the metric assume the familiar $d$-dimensional FLRW cosmologies as in (21). The evolution of the scale factor $a(t)$ then approaches a power law behaviour $a(t) \sim t^{\gamma}$, where the exponent $\gamma$ is related to the characteristic exponent $\alpha$ appearing in the potential. Combining the four-dimensional classification of [13] with the $d$-dimensional analysis of [14] (for $k=0$ ), one finds the attractor solutions given in table I. The different ranges of $\alpha^{2}$ are divided by the 'critical' and 'hypercritical' characteristic exponents

$$
\begin{aligned}
\alpha_{c}^{2} & =\frac{2}{d-2}, \\
\alpha_{h}^{2} & =\frac{2(d-1)}{d-2},
\end{aligned}
$$

respectively. The phase space analysis of [13] also shows that the $k=0$ solutions are unstable against perturbations in the spatial curvature of the universe.

Arbitrary amounts of accelerating expansion, or inflation, of the scale factor $a(t) \sim t^{\gamma}$, can only be obtained in two cases. In the standard case, the exponent is $\gamma>1$ (or equivalently, $\left.\alpha^{2}<\alpha_{c}^{2}\right)$ : the scale factor approaches an inflating attractor. The second case is the critical one where $\gamma=1$, which is realised for $\alpha^{2}=\alpha_{c}^{2}$ when $k=0$ and for $\alpha^{2} \geq \alpha_{c}^{2}$ when $k=-1$. Here, accelerating expansion can be achieved by choosing initial conditions such that the scalar fields start slowly rolling off the exponential potential. Later, the solution approaches 
the critical attractor, and, although tending to zero, the acceleration never stops. If, however, the attractor is a decelerating one, as for $\alpha^{2}>\alpha_{c}^{2}$ and $k=0$, then the approach of an actual solution, even setting initial conditions such that it starts off to be accelerating, to this attractor can produce only a short phase of accelerating expansion. It will quickly turn into decelerating expansion.

Now return to the case of many scalar fields $Q_{i}$ moving in a potential that is a sum of positive exponential terms, i.e., given by

$$
V\left(Q_{i}\right)=\sum_{i} \Lambda_{i} e^{-2 \sum_{j} \alpha_{i j} Q_{j}}
$$

where $\Lambda_{i}>0$. Consider the fields $Q_{i}$ to depend on time only, for an application to cosmological scenarios. Their time evolution is governed by their equations of motion and the Friedmann constraint which follows from the Einstein equations,

$$
\begin{aligned}
\ddot{Q}_{i}+(d-1) H \dot{Q}_{i}+\frac{\partial V}{\partial Q_{i}} & =0 \\
\alpha_{c}^{2} \sum_{i} \dot{Q}_{i}^{2}+2 \alpha_{c}^{2} V(\mathbf{Q})-k \alpha_{h}^{2} a^{-2} & =(d-1) H^{2},
\end{aligned}
$$

where the quantity $H=\dot{a} / a$ denotes the Hubble constant. This time evolution generically moves the scalar fields far away from the origin $\left(Q_{i}=0\right)$ in field space, at least when they are not trapped in a local minimum of the potential. This fact allows us to reformulate the problem of many scalar fields effectively in terms of a single one. At large norm squared in field space, i.e., for $\sum Q_{i}^{2} \gg 0$, it is only a single exponential term that dominates the potential $V\left(Q_{i}\right)$. To this dominating term, labelled by $i$, say, corresponds a direction of steepest descent of the potential, given by the vector $\mathbf{q}_{(i)}$ with components $\mathbf{q}_{(i) j}=\alpha_{i j}$, and the fields will approximately evolve into this gradient direction at late times. So going to infinity in field space along this direction, precisely as $\mathbf{Q}=|\mathbf{Q}| \frac{\mathbf{q}_{(i)}}{\left|\mathbf{q}_{(i)}\right|}$ with $|\mathbf{Q}| \rightarrow \infty$, provides effective characteristic exponents for an exponential potential of multiple scalar fields. Substituting the above relations,

$$
\Lambda_{i} e^{-2 \sum_{j} \alpha_{j i} Q_{j}} \sim \Lambda_{i} e^{-2\left|\mathbf{q}_{(i)}\right||\mathbf{Q}|}
$$

one finds their values to be

$$
\alpha_{i}^{2}=\left|\mathbf{q}_{(i)}\right|^{2}=\sum_{j} \alpha_{i j}^{2}
$$


This argument will be very useful later in discussing the possible types of attractor solutions occurring in product space compactifications.

This discussion of characteristic exponents for complicated scalar potentials also nicely complements the phase space analyses that have been done previously, albeit for simpler potentials. Special cases include, for instance, the 'cross-coupling' exponential potential, investigated in [15], where further references may be found.

\section{COMPACTIFICATIONS ON PRODUCT SPACES}

We set out, in this section, to study compactifications of $(d+n)$-dimensional gravity on product spaces. Extensions to include fluxes and dilaton fields appropriate to compactifications of string/M-theory will be studied in the following section IV where a part of the material produced here will be needed.

\section{A. Dimensional Reduction}

The action of $(d+n)$-dimensional pure Einstein gravity on a spacetime with metric $g_{M N}$ is completely determined by the Ricci scalar $R$ of $g_{M N}$,

$$
S_{(d+n)}=\int_{(d+n)} \sqrt{-g} R .
$$

The metric is split into its $d$-dimensional and its $n$-dimensional internal part according to

$$
d s^{2}=\tilde{g}_{\mu \nu}(x) d x^{\mu} d x^{\nu}+\hat{g}_{m n}(x, y) d y^{m} d y^{n},
$$

where the internal metric may also depend on the $d$-dimensional coordinates. This coordinate-dependence, however, is realised in a particular way. The internal space is taken to be a product of Einstein spaces $E_{p}(\Lambda)$, characterised by their dimension $p$ and a constant curvature $\Lambda$ that can have either sign or be zero. Each factor space is multiplied by an $x$-dependent function, so that its volume becomes $x$-dependent. These are the only moduli fields kept of the internal space metric. Otherwise, the factor Einstein spaces are assumed to be arbitrary but $d$-spacetime-independent. The sum of all dimensions adds to $\sum p_{i}=n$. Then the internal space metric and the Ricci tensor are block-diagonal and, in suitable 
conventions, they are given by

$$
\begin{aligned}
d \hat{s}^{2} & =\sum_{i} e^{2 F_{i}(x)} \hat{\omega}_{(i) \alpha \beta}(y) d y_{(i)}^{\alpha} d y_{(i)}^{\beta}, \\
\hat{R}_{(i) \alpha \beta} & =\Lambda_{i}\left(p_{i}-1\right) \hat{\omega}_{(i) \alpha \beta} .
\end{aligned}
$$

One clear advantage of such a configuration is that the Einstein equations with mixed indices in the $d$ - and $n$-dimensional spacetime parts, $R_{\mu m}=0$, are automatically satisfied. This is the case because $R_{\mu m}$ can be expressed in terms of $x$-derivatives of the internal space Christoffel symbols $\hat{\Gamma}_{m n}^{p}$, but these turn out to be $x$-independent.

Substituting the metric ansatz (11) into the $(d+n)$-dimensional action (10) causes a reduction down to $d$ dimensions (which is consistent as will be explained below),

$$
S_{d}=\int_{d} \sqrt{-\tilde{g}} e^{\Sigma}\left[\tilde{R}-2 \tilde{\square} \Sigma-(\tilde{\nabla} \Sigma)^{2}-\sum p_{i}\left(\tilde{\nabla} F_{i}\right)^{2}+\hat{R}\right],
$$

where $\Sigma(x)$ is defined as the sum of the volume moduli weighted by the dimension of their respective factor spaces,

$$
\Sigma(x)=\sum p_{i} F_{i}(x)
$$

Due to the special form of the internal space, its Ricci scalar depends only on $x$, as

$$
\hat{R}=\sum e^{-2 F_{i}} \Lambda_{i} p_{i}\left(p_{i}-1\right)
$$

Note also that the $x$-independent integral over the volume of the internal space,

$$
\int_{n} \prod \sqrt{\hat{\omega}_{(i)}}
$$

has been removed by an appropriate redefinition of the $d$-dimensional Newton constant. After dimensional reduction, however, the gravity part of $S_{d}$ is not in the standard EinsteinHilbert form: $\tilde{R}$ is multiplied by an additional function $e^{\Sigma}$. So in order to avoid possible interpretational problems with a spacetime-varying Newton constant, one has to remove $e^{\Sigma}$. This transformation into the Einstein frame is achieved by a conformal transformation of the metric $\tilde{g}_{\mu \nu}$. Note that such a conformal transformation leaves the causal structure of a spacetime unchanged but changes physical properties otherwise, as for example the acceleration of the scale factor, see also [1]. The Einstein frame metric $g_{E \mu \nu}$ is defined by

$$
g_{E \mu \nu}(x)=e^{\frac{2}{d-2} \Sigma(x)} \tilde{g}_{\mu \nu}(x)
$$


and transforming all terms of the action $S_{d}$ leads to the Einstein frame action

$$
S_{E}=\int_{d} \sqrt{-g_{E}}\left[R_{E}+\frac{2}{d-2} \square_{E} \Sigma-\frac{1}{d-2}\left(\nabla_{E} \Sigma\right)^{2}-\sum p_{i}\left(\nabla_{E} F_{i}\right)^{2}+\hat{R} e^{-\frac{2}{d-2} \Sigma}\right],
$$

where $\Sigma$ and $\hat{R}$ are given by (14) and (15), respectively. The surface term $\square_{E} \Sigma$ can now be dropped. This would not have been allowed before, in equation (13), since additional non-surface terms arise from it in the conformal transformation.

This compactification of the original $(d+n)$-dimensional action on the $n$-dimensional product space is consistent. This means that any solution of the equations of motion derived from $S_{E}$ (i.e., any solution of the Einstein equations with the appropriate scalar energymomentum and of the equations of motion for the volume scalars $F_{i}$ ) also solves the Einstein equations derived from $S_{(d+n)}$. Hence, any four-dimensional solution obtained can be lifted directly to higher dimensions.

\section{B. The Scalar Potential}

As shown in the previous subsection, the product space compactification (12) produces the four-dimensional action $S_{E}(18)$ in Einstein frame where gravity is coupled to the volume scalars $F_{i}$. In this action, however, the kinetic terms of the scalar fields are not in standard form, which, in our conventions, means that the system of gravity coupled to a single scalar $\phi$ should have the action (11). With an obviously unproblematic change of the overall normalisation, $S_{E}$ can be rewritten as

$$
S_{E}=\int_{d} \sqrt{-g_{E}}\left[\frac{1}{2} R_{E}-\nabla_{E} \mathbf{F}^{T} \mathcal{P} \nabla_{E} \mathbf{F}+\frac{1}{2} e^{-\frac{2}{d-2} \Sigma} \sum e^{-2 F_{i}} \Lambda_{i} p_{i}\left(p_{i}-1\right)\right],
$$

where $\mathbf{F}$ is the column vector formed from the fields $F_{i}$ and the matrix $\mathcal{P}$ is defined by its components

$$
\mathcal{P}_{i j}=\frac{1}{2(d-2)} p_{i} p_{j}+\frac{1}{2} p_{i} \delta_{i j}
$$

As $\mathcal{P}$ is symmetric, it can be diagonalised by an orthogonal matrix $\mathcal{S}$, satisfying $\mathcal{S S}^{T}=$ $\mathcal{S}^{T} \mathcal{S}=\mathbb{1}$, such that $\mathcal{S} \mathcal{P} \mathcal{S}^{T}=\operatorname{Diag}\left(P_{i}\right)$ where $P_{i}$ are the eigenvalues of $\mathcal{P}$. Several properties of $\mathcal{P}$ are needed for later calculations. The most important ones are

$$
\begin{aligned}
\operatorname{det} \mathcal{P} & =2^{-(M-1)}\left(1+\frac{1}{d-2} \sum p_{i}\right) \prod p_{j} \\
\left(\mathcal{P}^{-1}\right)_{i i} & =\frac{2}{p_{j}}-\frac{2}{d+n-2}
\end{aligned}
$$


where $M$ is the number of scalar fields $F_{i}$, i.e., the number of factor spaces of the internal product space. Defining new scalars $Q_{i}$ from the old ones $F_{i}$ by the invertible relation

$$
Q_{i} \equiv \sum_{j} \sqrt{P_{i}} \mathcal{S}_{i j} F_{j}
$$

the action is cast into the canonical form (henceforth the index ${ }_{E}$ denoting the Einstein frame will be dropped)

$$
S=\int_{d} \sqrt{-g}\left[\frac{1}{2} R-\nabla \mathbf{Q}^{T} \nabla \mathbf{Q}-2 V(\mathbf{Q})\right] .
$$

Expressed in the fields $Q_{i}$, the scalar potential is given by

$$
V(\mathbf{Q})=-\frac{1}{4} \sum_{i} \Lambda_{i} p_{i}\left(p_{i}-1\right) e^{-2 \frac{2}{p_{i}} \sum_{j} \sqrt{P_{j}} \mathcal{S}_{j i} Q_{j}}
$$

Positive contributions to the potential arise from negatively curved Einstein (factor) spaces where $\Lambda_{i}<0$ and vice versa.

Now assume the existence of an extremum of the potential at a point $\mathbf{Q}_{0}$ in field space where $\partial V / \partial Q_{i}=0$ for all values of $i$. A short calculation shows

$$
\sum_{i, j} \sqrt{P_{i}} \mathcal{S}_{i j} \frac{\partial V}{\partial Q_{i}}=-2\left(1+\frac{n}{d-2}\right) V .
$$

Hence, if there is an extremum of the potential (24), then it can only be at points $\mathbf{Q}_{0}$ where $V\left(\mathbf{Q}_{0}\right)=0$. This implies, in particular, that there is no positive extremum, and this fact will be of importance below. The non-existence of a positive extremum of the scalar potential in a supergravity compactification is also the essence of the no-go theorem [2].

\section{Effective Attractor Solutions}

Now that we have the precise form (24) of the potential of the canonically normalised scalar fields $Q_{i}$, we can make use of the general discussion of section II and calculate the corresponding characteristic exponents and effective attractor solutions.

Let all $N$ internal factors be Einstein spaces of zero or negative curvature. Then $V(\mathbf{Q}) \geq 0$ everywhere. This restriction serves a dual purpose. On the one hand, it excludes most scenarios with a big crunch that occurs whenever the left hand side in the Friedmann constraint (7b) becomes negative such that the evolution equations for the scalar fields break down 
(there still may be big crunches for the $k=+1$ cosmologies). On the other hand, this implies that the dominating terms in the potential are positive and then the above arguments for attractor solutions can be applied: a potential which is unbounded below does not allow for cosmological attractor solutions (as in the single scalar case).

If $V(\mathbf{Q}) \neq 0$ initially, then time evolution will always move the scalar fields to a large norm in field space, because the potential has no positive extremum, as we have seen. There exist up to $M$ directions $\left\{\mathbf{q}_{(i)} \mid k=1,2 \ldots\right\}$ of steepest descent of the potential, corresponding to the domination of a single exponential term far from the field space origin. These directions are read off from $V(\mathbf{Q})$ as

$$
q_{(i) j}=\frac{2}{p_{i}} \sqrt{P_{j}} \mathcal{S}_{j i},
$$

compare (6), and they determine the cosmological attractor solutions. According to (9), the characteristic exponents are given by

$$
\alpha_{i}^{2}=\sum_{j}\left(\frac{2}{p_{i}} \sqrt{P_{j}} \mathcal{S}_{j i}\right)^{2} .
$$

The question, of whether one gets large amounts of accelerating expansion, or inflation, from the product space compactification presented above, now reduces to a calculation of these characteristic exponents $\alpha_{i}$. From the definition of the matrix $\mathcal{P}$ in (20) and using the fact that it is positive definite, one finds

$$
\mathcal{P}_{i i}=\sum_{j}\left(\sqrt{P_{j}} \mathcal{S}_{j i}\right)^{2}=\frac{p_{i}}{2}\left(\frac{p_{i}}{d-2}+1\right),
$$

which can be rewritten to give the following simple formula:

$$
\alpha_{i}^{2}=\alpha_{c}^{2} \frac{p_{i}+d-2}{p_{i}}
$$

Note that the characteristic exponents always lie in the range between the critical one and the hypercritical one, $\alpha_{c}<\alpha_{i}<\alpha_{h}$ for $d>2$. The relevant attractor solutions in the potential $V(\mathcal{Q})$ (there are as many as there are internal Einstein spaces of strictly negative curvature) can be read off from table \ to be $a(t) \sim t$ for $k=-1$ and $a(t) \sim t^{\left(\alpha_{c} / \alpha_{i}\right)^{2}}$ for $k=0$. None of them is accelerating. So for $k=0$ one can at most obtain a short phase of accelerating expansion by choosing suitable initial conditions as explained in section II. This is the same mechanism as in most of the previously discussed exact solutions. In the $k=-1$ case, choosing the right initial conditions can lead to eternal acceleration. 
We emphasise that the important case of power law inflation, which would be implied by the existence of accelerating attractor solutions with $\alpha_{i}^{2} \leq \alpha_{c}^{2}$, is not realised in simple product space compactifications of pure gravity.

\section{STRING/M-THEORY COMPACTIFICATIONS}

To extend the application of the above results to product space compactifications of the various superstring theories or M-theory, one has to include the fields appearing in the corresponding low energy effective actions, as given by the ten and eleven-dimensional supergravities: antisymmetric field strengths $F_{p}$ with, possibly, dilaton couplings and the dilaton scalar field $\phi$ itself.

\section{A. Four Cases}

The new starting point is the $(d+n)$-dimensional bosonic action

$$
S_{(d+n)}=\int_{(d+n)} \sqrt{-g}\left[R-\frac{1}{2}(\nabla \phi)^{2}-\frac{1}{2 p !} e^{a \phi} F_{p}^{2}-\frac{1}{2} m^{2} e^{-\frac{5}{2} \phi}\right],
$$

where one might also want to include the mass term of massive IIA supergravity [16] by having $m \neq 0$. We do not consider possible Chern-Simons terms for the fluxes and their potentials here, as we will realise the fluxes by volume forms on certain subspaces below, which makes these terms irrelevant. After dimensional reduction down to $d$ dimensions, only a few different cases have to be considered. Compatible with the symmetries of the FLRW cosmologies, i.e., with the symmetries of a space with the topology $\mathbb{R} \times \Sigma_{k}$, there can exist the following field strength forms after compactification:

(i) $F_{0}$ and (ii) $F_{d}$,

(iii) $F_{1}$ with non-zero components only along the real time direction, and

(iv) $F_{d-1}$ with non-zero components only on the spatial sections $\Sigma_{k}$.

All other fluxes with non-negligible effects on cosmological scales are excluded (of course, this reasoning does not apply to local fields, for instance, electromagnetic fields in $d=4$ ).

In the dual cases (i) and (ii), an analysis of the $d$-dimensional equations of motion, for gravity, the field strengths and the scalars arising from the compactification process, shows that the field strengths $F_{0}$ and $F_{d}$ act as, and thus can be replaced by, genuine potentials 
for the scalar fields. They effectively become time-dependent cosmological constants. This becomes apparent, for example, in [4], where an $F_{4}$ arising from an M-theory compactification to four dimensions violates the four-dimensional strong energy condition by providing a positive scalar potential. (i) More explicitly, forms $F_{0}$ in $d$ dimensions arise from $F_{p}$ having components only in the internal space. We realise such $F_{p}$ as volume forms with field strength parameter $b_{0}$, i.e., we set $F_{p}=b_{0} v o l_{d, d+1 \ldots}$, and denote this type of reduction by

$$
F_{p}(d, d+1 \ldots) \rightsquigarrow F_{0}() .
$$

The reduced action in the $d$-dimensional Einstein frame then becomes

$$
S=\int_{d} \sqrt{-g}\left[\frac{1}{2} R-(\nabla \phi)^{2}-\nabla \mathbf{Q}^{T} \nabla \mathbf{Q}-2 V(\phi, \mathbf{Q})\right],
$$

and the scalar potential is given by

$$
V(\phi, \mathbf{Q})=V(\mathbf{Q})+\frac{1}{4} b_{0}^{2} e^{2 a \phi-\frac{2}{d-2} \Sigma(\mathbf{Q})-2 p_{1} F_{1}(\mathbf{Q})}+\frac{1}{8} m^{2} e^{-5 \phi-\frac{2}{d-2} \Sigma(\mathbf{Q})}, \quad p_{1}=p,
$$

where previous definitions (14), (22) and (24) have been used for $\Sigma$, the scalars $F_{i}(\mathbf{Q})$ and the potential term $V(\mathbf{Q})$, respectively. The original dilaton field $\phi$ has been canonically normalised by rescaling $\phi \mapsto 2 \phi$. The volume scalars $F_{i}$ should not be confused with field strengths. (ii) The reduction

$$
F_{p}(01 \ldots d-1 \mid d, \ldots) \rightsquigarrow F_{d}(01 \ldots d-1)
$$

gives the potential

$$
V(\phi, \mathbf{Q})=V(\mathbf{Q})+\frac{1}{4} b_{0}^{2} e^{-2 a \phi-\frac{2(d-1)}{d-2} \Sigma(\mathbf{Q})+2 p_{1} F_{1}(\mathbf{Q})}+\frac{1}{8} m^{2} e^{-5 \phi-\frac{2}{d-2} \Sigma(\mathbf{Q})}, \quad p_{1}=p-d .
$$

The cases (iii) and (iv) are also dual. Each field strength $F_{1}$ aligned along the time direction is equivalent to an additional scalar field $\psi$ via the relation $F_{1}=d \psi$, and spatial $(d-1)$-forms give rise to scalars via $\star F_{d-1}=F_{1}=d \psi$. The reduced action takes the form

$$
S=\int_{4} \sqrt{-g}\left[\frac{1}{2} R-(\nabla \phi)^{2}-\nabla \mathbf{Q}^{T} \nabla \mathbf{Q}-e^{C(\phi, \mathbf{Q})}(\nabla \psi)^{2}-2 V(\phi, \mathbf{Q})\right]
$$

(after normalising $\phi$ and $\psi$ ), where the function $C$ appears as a $d$-dimensional 'dilaton coupling'. (iii) For the reduction

$$
F_{p}(0 \mid d, d+1 \ldots) \rightsquigarrow F_{1}(0)
$$


one finds

$$
\begin{aligned}
C(\phi, \mathbf{Q}) & =2 a \phi-2 p_{1} F_{1}(\mathbf{Q}), \quad p_{1}=p-1 \\
V(\phi, \mathbf{Q}) & =V(\mathbf{Q})+\frac{1}{8} m^{2} e^{-5 \phi-\frac{2}{d-2} \Sigma(\mathbf{Q})}
\end{aligned}
$$

In case (iv) where

$$
F_{p}(1 \ldots d-1 \mid d \ldots) \rightsquigarrow F_{d-1}(1 \ldots d-1)
$$

one obtains

$$
\begin{aligned}
C(\phi, \mathbf{Q}) & =-2 a \phi-\frac{4}{d-2} \Sigma(\mathbf{Q})+2 p_{1} F_{1}(\mathbf{Q}), \quad p_{1}=p-d+1 \\
V(\phi, \mathbf{Q}) & =V(\mathbf{Q})+\frac{1}{8} m^{2} e^{-5 \phi-\frac{2}{d-2} \Sigma(\mathbf{Q})} .
\end{aligned}
$$

To answer the question whether inflation can be obtained, the same arguments as in the preceding sections can be applied. Note that (25) can easily be generalised. It is also true here that there do not exist any positive extrema of the potential. So we may look at dominant terms in the potential far from the origin in field space. Consider first the term arising from massive supergravity which is proportional to $e^{-2(5 \phi / 2+\Sigma /(d-2))}$. Without even taking the Q-dependence into account, one sees that the characteristic exponent $\alpha_{m}$ is larger than the critical one, $\alpha_{m}>\frac{5}{2}>\alpha_{c}$ for $d>2$.

In cases (i) and (ii), there is another term in the potential, arising from the field strength. The respective field space norms of the exponents can be calculated from

$$
\alpha_{F}^{2}=a^{2}+\frac{\zeta^{2}}{4} \sum_{i}\left(\sum_{j} \frac{p_{j}}{\sqrt{P_{i}}} \mathcal{S}_{i j}\left(1+\xi \delta_{j 1}\right)\right)^{2}
$$

where the parameters are given by $(\zeta, \xi)=\left(\alpha_{c}^{2}, 2 / \alpha_{c}^{2}\right)$ in case $(i)$ and $(\zeta, \xi)=\left(\alpha_{h}^{2},-2 / \alpha_{h}^{2}\right)$ in case (ii), respectively. The derivation uses the expression for the inverse of $\mathcal{P}$ in terms of its determinant and its adjoint. One finds

$$
\alpha_{F}^{2}=a^{2}+\frac{\zeta^{2}\left[-\xi^{2} p_{1}^{2}+((d+n-2) \xi+2(d-2)) \xi p_{1}+n(d-2)\right]}{2(d+n-2)}
$$

As an important example, look at string theory compactifications with $d=4$ and $n=6$. The value of the dilaton coupling is given by $a=\frac{1}{2}(5-p)$ where the degree $p$ of the field strength form is $p=p_{1}$ in case ( $i$ ) and $p=p_{1}-4$ in case (ii). An evaluation of the above expression then gives $\alpha_{F}^{2}=7$ independent of $p_{1}$, and for both cases. This is greater than 
the critical exponent $\alpha_{c}=1$. The conclusion, therefore, is that there are no accelerating attractor solutions for $k=0,+1$. For $k=-1$, one finds again the critical attractor with zero acceleration.

In cases (iii) and (iv) there are no further contributions to the potential. The difference here is a non-standard structure of the kinetic term of the field $\psi$, including a dilatoncoupling. (Such a coupling cannot be removed by simple field redefinitions.) Here, the above arguments might not be applicable in a straightforward way. It can be shown that actions with such non-standard scalar fields may admit power-law solutions quite different from those obtained when there are only scalars with standard kinetic terms. This happens, for example, in the model

$$
\begin{aligned}
S=\int \sqrt{-g}\left[\frac{1}{2} R-(\nabla \phi)^{2}-(\nabla Q)^{2}-e^{-2\left(a_{0} \phi+a_{1} Q\right)}(\nabla \psi)^{2}\right. \\
\left.-2 \Lambda e^{-2 \alpha \phi}-\frac{1}{4} M^{2} e^{-2\left(a_{2} \phi+a_{3} Q\right)}\right]
\end{aligned}
$$

that arises as a special case of both, (iii) and (iv). But, in these cases, the scale factor $a(t) \sim t^{\gamma}$ never has a solution with $\gamma>1$. So the non-standard coupling of the scalar fields does not make it easier to find accelerated expansion. This is, in fact, to be expected because these terms provide additional kinetic energy and make it harder to satisfy the acceleration condition (3).

\section{B. Scale Invariance}

The supergravity actions including a dilaton field have a scale invariance which survives the compactification process. As was noted in [17], the scalar potential $V\left(\phi, Q_{i}\right)$ in the compactified theory can then be written as a product. Identify $Q_{0} \equiv \phi$. We expect that

$$
V\left(Q_{\mu}\right)=V\left(Q_{0}\right) V\left(Q_{i}\right)
$$

and that the dependence of $V\left(Q_{0}\right)$ on $Q_{0}$ is purely exponential. To see that this is the case, note that the $(M+1)$ scalar fields $Q_{\mu}$, arising from the original higher-dimensional dilaton and from the volume moduli of the internal space, are only defined up to an $S O(M+1)$ rotation, since this leaves their kinetic terms invariant. To achieve a product decomposition of the potential, one generically has to perform such a rotation. 
We will now construct this rotation explicitly for the $F_{p}(d, d+1 \ldots) \rightsquigarrow F_{0}()$ and $F_{p}(01 \ldots d-$ $1 \mid d \ldots) \rightsquigarrow F_{d}(01 \ldots d-1)$ reductions, respectively (setting the mass parameter $m$ to zero). We define new fields $\widetilde{Q}_{\nu}$, rotating by a matrix $\mathcal{T} \in S O(M+1)$, as

$$
Q_{\mu} \equiv \sum_{v} \mathcal{T}_{\mu \nu} \widetilde{Q}_{\nu}
$$

The scalar potential is given from (33) or, respectively, (35) as

$$
\begin{aligned}
V\left(\widetilde{Q}_{\nu}\right)= & -\frac{1}{4} \sum_{k} \Lambda_{k} p_{k}\left(p_{k}-1\right) e^{-2 \sum_{\nu}\left(\sum_{j} \frac{2}{p_{k}} \sqrt{P_{j}} \mathcal{S}_{j k} \mathcal{T}_{j \nu}\right) \widetilde{Q}_{\nu}} \\
& +\frac{1}{4} b_{0}^{2} e^{-2 \sum_{\nu}\left(\mp a \mathcal{T}_{0 \nu}+\zeta \sum_{i j} \frac{p_{i}}{2 \sqrt{P_{j}}} \mathcal{S}_{j i}\left(1+\xi \delta_{i 1}\right) \mathcal{T}_{j \nu}\right) \widetilde{Q}_{\nu}}
\end{aligned}
$$

where we use the parameters $(\zeta, \xi)=\left(\alpha_{c}^{2}, 2 / \alpha_{c}^{2}\right)$ and the upper sign for $F_{p}(d, d+1 \ldots) \rightsquigarrow F_{0}()$, and $(\zeta, \xi)=\left(\alpha_{h}^{2},-2 / \alpha_{h}^{2}\right)$ and the lower sign, for the potential from the $F_{p}(01 \ldots d-1 \mid d \ldots) \rightsquigarrow$ $F_{d}(01 \ldots d-1)$ reduction. To split off, from the potential, a common factor of the form $e^{-2(\ldots) \widetilde{Q}_{0}}$, the condition

$$
\pm a \mathcal{T}_{00}=\sum_{j}\left(\zeta \sum_{i} \frac{p_{i}}{2 \sqrt{P_{j}}} \mathcal{S}_{j i}\left(1+\xi \delta_{i 1}\right)-\frac{2}{p_{k}} \sqrt{P_{j}} \mathcal{S}_{j k}\right) \mathcal{T}_{j 0}
$$

has to be satisfied for all values of $k=1, \ldots, M-1$. This can be done by setting

$$
\begin{aligned}
& \mathcal{T}_{00}= \pm \frac{\left(\zeta-\alpha_{c}^{2}\right) n+\zeta \xi p_{1}-2}{2 a} \gamma, \\
& \mathcal{T}_{j 0}=\sum_{l} \sqrt{P_{j}} \mathcal{S}_{j l} \gamma .
\end{aligned}
$$

The normalisation constant $\gamma$ ensures that $\mathcal{T}_{\mu 0}$ are components of an $S O(M+1)$-matrix. The condition is $\sum_{\mu} \mathcal{T}_{\mu 0}^{2}=1$ and is satisfied for

$$
\gamma^{2}=4 a^{2}\left[\left(\left(\zeta-\alpha_{c}^{2}\right) n+\zeta \xi p_{1}-2\right)^{2}+a^{2} \alpha_{c}^{2} n(d+n-2)\right]^{-1} .
$$

Now that we have shown that the potential can be written in product form, it is interesting to check the characteristic exponent of the factor $V\left(\widetilde{Q}_{0}\right)$. It is $\alpha_{0}=\frac{d+n-2}{d-2} \gamma$ and will be subcritical, $\alpha_{0}^{2} \leq \alpha_{c}^{2}$, if, and only if,

$$
\left(\left(\zeta-\alpha_{c}^{2}\right) n+\zeta \xi p_{1}-2\right)^{2} \geq a^{2} \alpha_{c}^{2}(d+n-2)\left(2-n \frac{d-4}{d-2}\right) .
$$

For string theory compactifications with $d=4$ and $n=6$, the value of the dilaton coupling is $a=\frac{1}{2}(5-p)$ where the degree $p$ of the field strength form is $p=p_{1}$ in the first case and 
$p=p_{1}-4$ in the second. One checks that the exponent is subcritical for $p=p_{1}>2$ or, in the second case, for $p-4=p_{1} \leq 3$ (exactly critical for $p=7$ ).

This is potentially interesting because one might imagine the potential having a flat direction, becoming constant near infinity, in the $M$-dimensional field space of the $\widetilde{Q}_{i}$. Then choosing initial conditions such that all fields start at rest in this region, they would evolve towards an accelerating attractor in direction of $\widetilde{Q}_{0}$, while at the same time rolling very slowly off the almost flat potential in the other directions. This might yield a large amount of inflation and would at the same time provide a mechanism to stop the inflationary epoch. To look for such flat directions note that, by the same argument as employed above, a single exponential term of the potential factor $V\left(\widetilde{Q}_{i}\right)$ dominates at large $|\widetilde{\mathbf{Q}}|$. It is of the form $e^{-2 \widetilde{\alpha} \cdot \widetilde{\mathbf{Q}}}$ and constant if, and only if, $|\widetilde{\alpha}|^{2}=0$. The expression $V\left(\widetilde{Q}_{i}\right)$ is given by (46) with the only difference that, in the exponents, one has to sum only over $i$, i.e., over $\nu \neq 0$. We calculate the characteristic exponents $|\widetilde{\alpha}|^{2}$ for all exponential terms in turn. For those in the sum over the curvatures $\Lambda_{k}$ of the internal space factors, we find

$$
\widetilde{\alpha}_{k}^{2}=\frac{2}{d-2}+\frac{2}{p_{k}}-\gamma^{2} \frac{(d+n-2)^{2}}{(d-2)^{2}} .
$$

For the term arising from the field strength, using $\alpha_{F}^{2}$ from (42), one obtains

$$
\widetilde{\alpha}_{F}^{2}=\alpha_{F}^{2}-\frac{1}{4} \gamma^{2}\left(\alpha_{c}^{2} n+2\right)^{2} .
$$

For string theory compactifications down to four dimensions, these expressions never become zero. Thus the potential does not have flat directions in $\widetilde{Q}_{i}$-space.

The arguments of this section also hold if one includes the mass term of IIA supergravity but sets the field strength to zero. The calculation proceeds as before. For the parameters one has to substitute $a=-\frac{5}{2}$ and $(\zeta, \xi)=(1,0)$. For $d=4$ and $n=6$, this case also allows a factorisation of the potential into a pure exponential with characteristic exponent $\alpha_{0}>1$, and into another factor that does not have any flat directions either.

\section{CONSISTENT TRUNCATIONS}

We have seen that the product space compactification produces characteristic exponents that are always above the critical value $\alpha_{c}$. As discussed above, this results in the nonexistence of solutions with genuine power-law inflation. This section presents a mechanism of reducing the characteristic exponents by truncating the number of scalar fields. 
Suppose the compactification produces two scalar fields $\phi$ and $Q$. In certain circumstances, it is then possible to truncate the compactified theory consistently, such that only one scalar field $\psi$ is left. This is performed by choosing a certain direction in the originally two-dimensional field space. Set

$$
\phi=\frac{s}{\sqrt{1+\lambda^{2}}} \psi \quad \text { and } \quad Q=\frac{\lambda}{\sqrt{1+\lambda^{2}}} \psi
$$

with $s^{2}=1$ and an at first arbitrary parameter $\lambda$ to be determined later. (This procedure can be generalised to a higher number of fields but becomes increasingly messy.) To see that such a truncation is interesting and reduces the characteristic exponent in a contribution to the potential, consider a typical term proportional to $e^{-2(\alpha \phi+\beta Q)}$. The characteristic exponent, before the truncation, is $\alpha_{0}^{2}=\alpha^{2}+\beta^{2}$. After the truncation, one finds

$$
\alpha_{\text {trunc. }}^{2}=\frac{\alpha^{2}+\beta^{2} \lambda^{2}+2 \alpha \beta s \lambda}{1+\lambda^{2}} .
$$

This expression is smaller than $\alpha_{0}^{2}$, unless $\alpha s \lambda-\beta=0$. So, generically, a consistent truncation reduces the characteristic exponents, and thus it presents another, possibly helpful mechanism of generating accelerating expansion.

To demonstrate this concept, we consider a reduction of the type

$$
F_{4}(0123) \rightsquigarrow F_{4}(0123)
$$

in a $(4+n)$-dimensional theory. The internal space shall only have a single factor and we set $m=0$. In terms of the fields $\phi$ and $Q$, the potential (35) becomes

$$
V(\phi, Q)=-\frac{1}{4} \Lambda n(n-1) e^{-2 \sqrt{\frac{n+2}{n}} Q}+\frac{1}{4} b_{0}^{2} e^{-2 a \phi-6 \sqrt{\frac{n}{n+2}} Q} .
$$

The consistency condition for the truncation is that both equations of motion for the scalar fields are solved simultaneously when substituting (5.53). This determines

$$
s \lambda=-\frac{a \sqrt{n(n+2)}}{2(n-1)}
$$

and

$$
\frac{1}{2} \Lambda(n-1)=\frac{1}{2} b_{0}^{2}\left(\frac{a^{2}}{2(n-1)}+\frac{3}{n+2}\right) .
$$

Calculating the characteristic exponent $\alpha_{\text {trunc. }}^{2}$ using (54) with $\alpha=0$ and $\beta=\sqrt{\frac{n+2}{n}}$, one finds that it is in fact smaller than $\alpha_{c}=1$ (for $n=6$ and $a=\frac{1}{2}$, appropriate to a 
compactification of IIA superstring theory). But the potential of the truncated theory turns out to be negative, such that there cannot be accelerating attractor solutions! We would have needed a positive potential.

So far no example has been found, where a consistent truncation leads to a theory that admits solutions with an arbitrary amount of accelerating expansion, by providing an accelerating power-law attractor for the FLRW scale factor.

\section{DISCUSSION}

Starting from the Einstein-Hilbert action for pure gravity in $(d+n)$ dimensions, and also from the bosonic part of various supergravity actions that describe string/M-theory in the low energy limit, a dimensional reduction down to $d$ dimensions has been performed. The $n$ dimensional internal space was realised as a product space, and the moduli fields kept in this reduction determined the volume of each of the factor spaces. Otherwise these factor spaces have been considered to be arbitrary but fixed $d$-independent Einstein spaces. The effective $d$-dimensional field theory has been studied in Einstein conformal frame in order to facilitate an easy interpretation of the gravity results from the lower dimensional point of view. The main focus of this paper was the investigation of the potential for the scalar fields, the volume moduli, resulting from the compactification. We were interested in cosmological applications, not so much in phenomenological aspects but rather in a question of principle: whether it is possible to find FLRW solutions, from gravity or string/M-theory compactifications, in which the scale factor exhibits arbitrary amounts of accelerating expansion, or inflation.

We have argued that the discussion for the case of multiple scalar fields can be understood effectively in terms of a single scalar field. The time evolution essentially picks out, far from the origin in field space, a dominant direction of the potential. The sum of various exponentials thereby is reduced to a single effective exponential term. For this term, it is possible to calculate a characteristic exponent that directly classifies the existing attractor solutions for the scale factor according to table [1] In this way, the intuition from the single scalar case can be carried over to the more complicated case.

The calculation of the characteristic exponents for the potentials, arising from the $n$ dimensional geometry and from various fluxes that were realised as volume forms on certain subspaces, revealed that they are always greater than the critical exponent in $d$-dimensions. 
The conclusions drawn from this fact depend on the possible values $k=0, \pm 1$ of the curvature of the spatial sections in the final cosmology. In the flat case with $k=0$, this means that there do not exist any accelerating attractor solutions for the scale factor. The only way in which accelerating expansion can be obtained is via the mechanism in the original solutions (subsequent to [1], see the introduction) as identified in [7]. Any such solution can only have a short phase of accelerating expansion, where the scalar fields are nearly at rest at some point in field space, around which the potential dominates. But this phase ends soon as the decelerating attractor is approached. (Note that we do not claim that there is no way to improve on the $\mathcal{O}(1)$ expansion in these scenarios. But the generic existence of decelerating attractors does make it extremely implausible to achieve sixty e-foldings). For hyperbolic spatial curvature $k=-1$, characteristic exponents greater than $\alpha_{c}$ mean that the attractors are exactly critical and non-accelerating, $a(t) \sim t$. In this case one can obtain eternal accelerating expansion. This happens in solutions in which trajectories exist with a point where the scalar fields are approximately at rest (as above). Then the scale factor starts off accelerating and, as it approaches the critical attractor, this acceleration does not stop although it tends to zero. Such a scenario has been identified in [11] by perturbing around the attractor solution. We have not considered the case $k=+1$ because it does not admit attractor solutions. At best, solutions may behave, for a while, in a similar way as do the $k=0$ ones, as suggested by the analysis of [13], but they all end in a big crunch.

In summary, it has been shown that product space compactifications do not lead to standard inflationary scenarios (with power-law inflation of the scale factor). Cosmological solutions in this setup do only admit a short phase of accelerating expansion for $k=0$ (and $k=+1$ ). For $k=-1$, the generic solution can lead to eternal accelerating expansion, but the acceleration quickly tends to zero. Thus, in order to obtain inflation from higher dimensions and, in particular, from string/M-theory, it seems to be necessary to think about more complex scenarios of dimensional reduction. Possibilities of improvement are the inclusion of further warp factors, possibly the mechanism of consistent truncation presented in the preceding section, or generically more complicated internal space geometries. It might also be necessary to consider non-trivial contributions due to Chern-Simons terms in the supergravity actions. We leave these avenues for future research. 


\section{Acknowledgments}

MNRW thanks Paul K. Townsend for very helpful discussions, and also Nobuyoshi Ohta and John E. Wang. He gratefully acknowledges financial support from the Gates Cambridge Trust and wishes to thank Trinity College Cambridge and the organisers of Strings 2003 for enabling him to visit this conference in Kyoto.

[1] P.K. Townsend and M.N.R. Wohlfarth, Accelerating cosmologies from compactification, to be publ. in Phys. Rev. Lett., [hep-th/0303097].

[2] G.W. Gibbons, Aspects of supergravity theories in Supersymmetry, supergravity and related topics, edited by F. del Aguila, J.A. de Azcárraga and L. Ibáñez, 123 (World Scientific, Singapore, 1985); J. Maldacena and C. Nuñez, Supergravity description of field theories on curved manifolds, and a no go theorem, Int. J. Mod. Phys. A16 (2001) 822.

[3] N. Ohta, Accelerating cosmologies from S-branes, to be publ. in Phys. Rev. Lett., [hep-th/0303238]; S. Roy, Accelerating cosmologies from M/string theory compactifications, to be publ. in Phys. Lett. B, [hep-th/0304084].

[4] M.N.R. Wohlfarth, Accelerating cosmologies and a phase transition in M-theory, Phys. Lett. B563 (2003) 1.

[5] N. Ohta, A study of accelerating cosmologies from superstring/M-theories, to be publ. in Prog. Theor. Phys., [hep-th/0304172].

[6] M. Gutperle, R. Kallosh and A. Linde, M/string theory, S-branes and accelerating universe, [hep-th/0304225].

[7] R. Emparan and J. Garriga, A note on accelerating cosmologies from compactifications and S-branes, JHEP 05 (2003) 028.

[8] G.W. Gibbons, Phantom matter and the cosmological constant, [hep-th/0302199]; P. Singh, M. Sami and N. Dadhich, Cosmological dynamics of phantom field, to be publ. in Phys. Rev. D, [hep-th/0305110]; M.P. Dabrowski, T. Stachowiak and M. Szydlowski, Phantom Cosmologies, [hep-th/0307128].

[9] C.M. Hull, Timelike T-duality, de Sitter space, large $N$ gauge theories and topological field theory, JHEP 07 (1998) 021. 
[10] C.-M. Chen, P.-M. Ho, I.P. Neupane and J.E. Wang, A note on acceleration from product space compactification, JHEP 07 (2003) 017.

[11] C.-M. Chen, P.-M. Ho, I.P. Neupane, N. Ohta and J.E. Wang, Hyperbolic space cosmologies, [hep-th/0306291].

[12] E.J. Copeland, A.R. Liddle and D. Wands, Exponential potentials and cosmological scaling solutions, Phys. Rev. D57 (1998) 4686; I.P.C. Heard and D. Wands, Cosmology with positive and negative exponential potentials, Class. Quant. Grav. 19 (2002) 5435.

[13] J.J. Halliwell, Scalar fields in cosmology with an exponential potential, Phys. Lett. B185 (1987) 341.

[14] P.K. Townsend, Quintessence from M-theory, JHEP 11 (2001) 042.

[15] Z.-K. Guo, Y.-S. Piao, R.-G. Cai and Y.-Z. Zhang, Cosmological scaling solutions and crosscoupling exponential potential, [hep-th/0306245].

[16] L.J. Romans, Massive $N=2 A$ supergravity in ten dimensions, Phys. Lett. B169 (1986) 374.

[17] G.W. Gibbons and P.K. Townsend, Cosmological evolution of degenerate vacua, Nucl. Phys. B282 (1987) 610. 